\title{
Integrated slice-specific dynamic shimming diffusion weighted imaging (DWI) for rectal Cancer detection and characterization
}

Jianxing Qiu ${ }^{1 \dagger}$, Jing Liư ${ }^{1 \dagger}$, Zhongxu Bi ${ }^{1}$, Xiaowei Sun ${ }^{1}$, Xin Wang ${ }^{2}$, Junling Zhang ${ }^{2}$, Chengwen Liu ${ }^{3}$, Jinxia Zhu ${ }^{3}$ and Naishan Qin ${ }^{1 *}$

\begin{abstract}
Purpose: To compare integrated slice-specific dynamic shimming (iShim) diffusion weighted imaging (DWI) and single-shot echo-planar imaging (SS-EPI) DWI in image quality and pathological characterization of rectal cancer.

Materials and methods: A total of 193 consecutive rectal tumor patients were enrolled for retrospective analysis. Among them, 101 patients underwent iShim-DWI $\left(b=0,800\right.$, and $\left.1600 \mathrm{~s} / \mathrm{mm}^{2}\right)$ and 92 patients underwent SS-EPIDWI $\left(b=0\right.$, and $\left.1000 \mathrm{~s} / \mathrm{mm}^{2}\right)$. Qualitative analyses of both DWI techniques was performed by two independent readers; including adequate fat suppression, the presence of artifacts and image quality. Quantitative analysis was performed by calculating standard deviation (SD) of the gluteus maximus, signal intensity (SI) of lesion and residual normal rectal wall, apparent diffusion coefficient (ADC) values (generated by b values of 0,800 and $1600 \mathrm{~s} / \mathrm{mm}^{2}$ for iShim-DWI, and by $b$ values of 0 and $1000 \mathrm{~s} / \mathrm{mm}^{2}$ for SS-EPI-DWI) and image quality parameters, such as signal-tonoise ratio (SNR) and contrast-to-noise ratio (CNR) of primary rectal tumor. For the primary rectal cancer, two pathological groups were divided according to pathological results: Group 1 (well-differentiated) and Group 2 (poorly differentiated). Statistical analyses were performed with $p<0.05$ as significant difference.

Results: Compared with SS-EPI-DWI, significantly higher scores of image quality were obtained in iShim-DWI cases $(P<0.001)$. The $\mathrm{SD}_{\text {background }}$ was significantly reduced on $b=1600 \mathrm{~s} / \mathrm{mm}^{2}$ images and ADC maps of iShim-DWl. Both SNR and CNR of $b=800 \mathrm{~s} / \mathrm{mm}^{2}$ and $b=1600 \mathrm{~s} / \mathrm{mm}^{2}$ images in iShim-DWI were higher than those of $b=1000 \mathrm{~s} / \mathrm{mm}^{2}$ images in SS-EPI-DWI. In primary rectal cancer of iShim-DWI cohort, Sl lesion was significantly higher than $\mathrm{SI}_{\text {rectum }}$ in both $\mathrm{b}=800$ and $1600 \mathrm{~s} / \mathrm{mm}^{2}$ images. ADC values were significantly lower in Group $2(0.732 \pm$ $\left.0.08) \times 10^{-3} \mathrm{~mm}^{2} / \mathrm{s}\right)$ than those in Group $1\left((0.912 \pm 0.21) \times 10^{-3} \mathrm{~mm}^{2} / \mathrm{s}\right)$. ROC analyses showed significance of ADC values and $\mathrm{Sl}_{\text {lesion }}$ between the two groups.
\end{abstract}

Conclusion: iShim-DWI with b values of 0,800 and $1600 \mathrm{~s} / \mathrm{mm}^{2}$ is a promising technique of high image quality in rectal tumor imaging, and has potential ability to differentiate rectal cancer from normal wall and predicting pathological characterization.

Keywords: Rectal Cancer, Integrated slice-specific dynamic shimming (iShim); diffusion weighted imaging (DWI), Imaging quality, Tumor differentiation

\footnotetext{
* Correspondence: Qinnaishan@bjmu.edu.cn

${ }^{\dagger}$ Jianxing Qiu and Jing Liu contributed equally to this work.

'Department of Radiology, Peking University First Hospital, 8 XishiKu Avenue, XiCheng District, Beijing 100034, China

Full list of author information is available at the end of the article
}

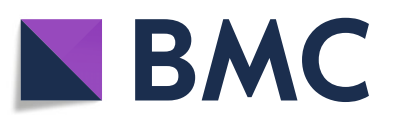

(- The Author(s). 2021 Open Access This article is licensed under a Creative Commons Attribution 4.0 International License, which permits use, sharing, adaptation, distribution and reproduction in any medium or format, as long as you give appropriate credit to the original author(s) and the source, provide a link to the Creative Commons licence, and indicate if changes were made. The images or other third party material in this article are included in the article's Creative Commons licence, unless indicated otherwise in a credit line to the material. If material is not included in the article's Creative Commons licence and your intended use is not permitted by statutory regulation or exceeds the permitted use, you will need to obtain permission directly from the copyright holder. To view a copy of this licence, visit http://creativecommons.org/licenses/by/4.0/. The Creative Commons Public Domain Dedication waiver (http://creativecommons.org/publicdomain/zero/1.0/) applies to the data made available in this article, unless otherwise stated in a credit line to the data. 


\section{Key results}

1. iShim-DWI improves images quality of rectal cancer compared with SS-EPI-DWI.

2. ADC Values generated by $b$ values of 0,800 and $1600 \mathrm{~s} / \mathrm{mm}^{2}$ in iShim-DWI can help differentiate high-grade cancer from low-grade cancer.

\section{Introduction}

Diffusion-weighted imaging (DWI) is increasingly used for detection and characterization of rectal cancer in clinical practice [1-5]. Single-shot echo-planar imaging (SS-EPI) DWI has been routinely used as the main DWI sequence. However, SS-EPI-DWI has limited spatial resolution and is sensitive to motion and magnetic field inhomogeneity [6, 7]. In addition, susceptibility artifacts from gas in rectum may also cause image distortion. Advancement in DWI technology makes it possible to perform DWI with ultrahigh $b$ value or multiple $b$-values to obtain more information for lesion detection and characterization [8-12]. The most widely used high b-values for DWI in rectal cancer are 800 and $1000 \mathrm{~s} / \mathrm{mm}^{2}$ in routine work [11, 13-15]. Lower b-values are always associated with a risk of 'T2shine-through' effects. DWI with ultra-high b-values (above $1000 \mathrm{~s} / \mathrm{mm} 2$ ) might allow for a better visualization of tumors due to a highly effective suppression of background signal [9-12]. A previous study using DWI with $b$ value of $2000 \mathrm{~s} / \mathrm{mm}^{2}$ in rectal cancer imaging suggested that signal intensity (SI) helps assess the primary rectal tumor and its response to chemo-radiation therapy (CRT) [10]. However, the resolution of images with ultra-high $\mathrm{b}$ value is limited for clinical diagnosis. A solution is to combine high and ultra-high $b$ values to balance spatial resolution and functional information. Chen et al., [11] investigated different bvalue combination and suggested the apparent diffusion coefficient (ADC) value generated by combining high and ultra-high $\mathrm{b}$ values shows improvement in rectal cancer characterization in 32 patients.

Recently, integrated slice-specific dynamic shimming (iShim) DWI has been reported to improve image quality and lesion detection in bladder, prostate, breast, and thyroid compared with SS-EPI-DWI [16-20]. In this study, we investigate the performance of iShim-DWI in detection and characterization of rectal cancer, in comparison with SS-EPI-DWI technology.

\section{Material and methods}

\section{Ethics statement}

This study was approved by institutional review board. The need for written informed consent was waived by the institutional review board due to the retrospective design of the study.

\section{Patients enrollment}

A total of 204 consecutive patients with suspected rectal cancer by surgeon or histologically proven rectal cancer were referred for a multiparametric MR examination from June 2018 to June 2020. Among them, 2 patients received both SS-EPI-DWI and iShim-DWI to testify the feasibility of iShim-DWI.

Among the rest 202 patients, 107 consecutive cases were imaged with iShim-DWI, and 95 consecutive cases with SS-EPI-DWI. Consecutive patients were selected for each cohort. The inclusion criteria for rectal cancer were as follows: proven rectal tumor by either biopsy or surgery (including both primary tumor and after treatment). The exclusion criteria were as follows: non-rectal tumor confirmed by endoscopy at the first time.

Among the 107 iShim-DWI cases, 6 cases were excluded due to ulcerative colitis (3), rectal inflammation with unknown reason (2), and rectum invaded by fallopian tube carcinoma (1). Among the SS-EPI-DWI cases, 3 patients were excluded due to epidermoid cyst (1),

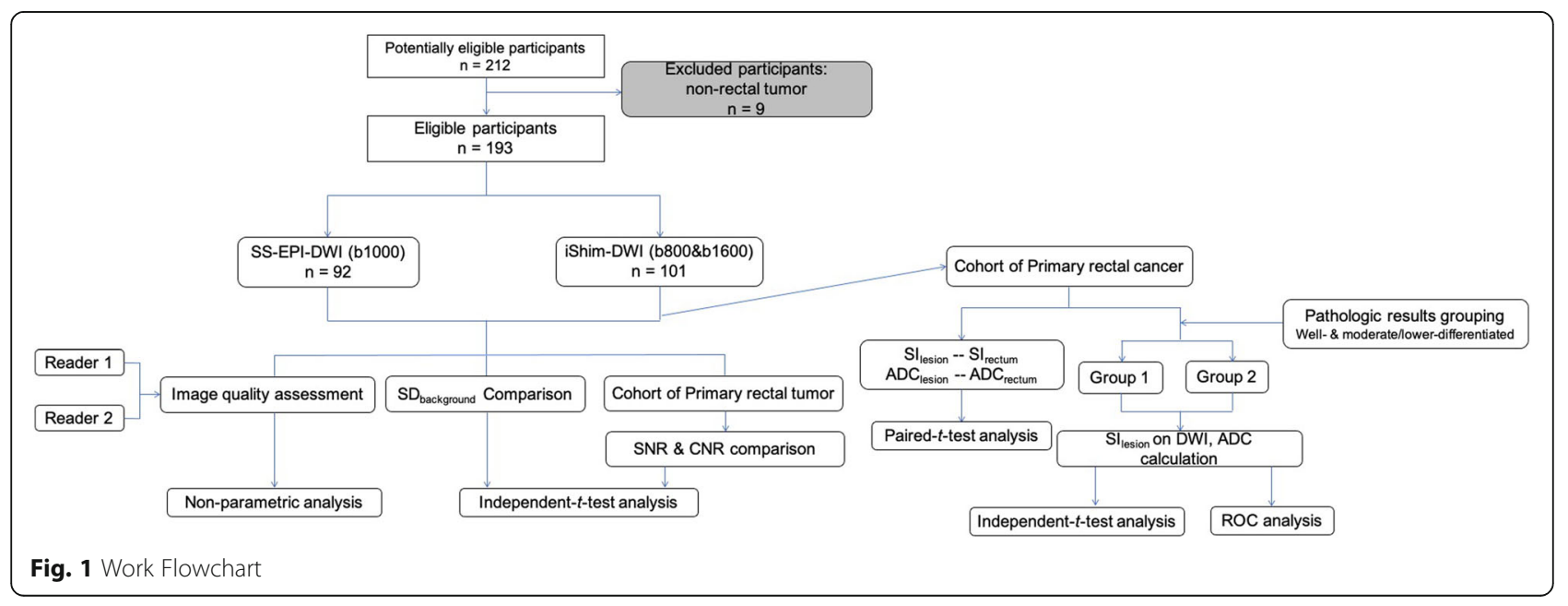


intussusception (1), and radiation enteritis from cervical carcinoma (1) (Fig. 1).

\section{MRI acquisition}

All MRI examinations were performed on a MAGN ETOM Aera 1.5 T MRI scanner (Siemens Healthcare, Erlangen, Germany) with an 18-channel body coil and a 32-channel spine coil. No special bowel preparation was applied. The rectal cancer examination protocols included T2-weighted Turbo Spin Echo (T2W TSE) images in axial, sagittal, coronal orientation, and DWI using a prototype iShim-DWI (Siemens Healthcare, Erlangen, Germany) or standard SS-EPI-DWI. ADC maps were calculated inline for each DWI sequence with a monoexponentially fit based on the 3 or 2 measured b-values. Then ten dynamic enhanced T1W images were collected after administering body weight-adapted intravenous gadopentetate dimeglumine $(0.1 \mathrm{mmol} / \mathrm{kg}$ BW, Dotarem; Guerbet, Paris, France) at a rate of $2 \mathrm{~mL} / \mathrm{s}$. Protocol parameters for these sequences were summarized in Supplement 1.

\section{Qualitative assessment}

Images were interpreted using the Picture Archiving and Communication Systems (PACS) workstations. Two experienced radiologists (Reader 1 with 10 years and Reader 2 with 20 years of experience in abdominal MRI) who were unaware of patients' clinical data assessed DWI images independently, including both iShim DWI and SS-EPI-DWI, and rated the image quality of DWI images (b1000 for SS-EPI-DWI, both $b=800$ and 1600 $\mathrm{s} / \mathrm{mm}^{2}$ for iShim-DWI), ADC maps for each patient according to a Likert-type scale:

1) Adequate fat suppression for DW images:

- Score 1: failure in suppression;

- Score 2: regional fat-water failures but still interpretable;

- Score 3: minimal failures in image periphery;

- Score 4: perfect fat-water separation

2) Presence of artifact or artifacts for both DW images and ADC maps:

- Score 1: nondiagnostic;

- Score 2: artifacts but diagnostic;
- Scroe 3: no artifact.

3) Image quality for both DW images and ADC maps:

- Score 1: nondiagnostic image quality with serious artifacts, image distortion, or poor signal intensity.

- Score 2: poor diagnostic image quality and diagnostic confidence of the readers, with serious artifacts, image distortion pronounced, or relatively poor signal intensity.

- Score 3: moderate image quality and confidence of the readers: with some artifacts, moderate image distortion.

- Score 4: good diagnostic image quality and confidence of the readers: with few artifacts, slight image distortion, anatomic structures (e.g. rectal wall) well delineated.

- Score 5: excellent image quality and strong confidence of the readers in the diagnosis: with almost no artifacts or imaging distortion.

The 2 readers assessed the images separately in a blinded, randomized fashion. The discrepancies were resolved in a consensus reading. The results of the consensus reading were used for statistical analyses.

\section{Quantitative assessment}

Reader 1 calculated the SI on DW images and ADC values on ADC maps in all the patients using PACS.

For ADC maps:

1. Two region of interests (ROIs) of no less than 20 $\mathrm{mm}^{2}$ were placed on different section of the lesion. The ROIs were manually drawn tracing along the margin of the lesion on ADC map, and to maximumly include the whole solid part of lesion with avoiding necrosis and hemorrhage. The average value caculated by the two ROIs were regarded as the mean $\mathrm{ADC}_{\text {lesion. }}$.

2. Two ROIs of nearly $10 \mathrm{~mm}^{2}$ were placed on different part of the residual normal rectum. The average values caculated by the two were regarded as the mean $\mathrm{ADC}_{\text {rectum. }}$.

3. One ROI of $100 \mathrm{~mm}^{2}$ was placed on the right gluteus maximus. The standard deviation (SD) was regarded as the background noise: $\mathrm{SD}_{\text {background-ADC }}$ for ADC map.

For DW Images: 
1. Two ROIs of no less than $20 \mathrm{~mm}^{2}$ were placed in the lesion (for SS-EPI-DWI, drawn on images of $\mathrm{b}=1000 \mathrm{~s} / \mathrm{mm}^{2}$, and for iShim-DWI, drawn on both images of $b=800$ and $1600 \mathrm{~s} / \mathrm{mm}^{2}$ ) with the same location of ADC maps. The average values caculated by the two ROIs were regarded as $\mathrm{SI}_{\text {lesion }}$ separetely.

2. Two ROIs of nearly $10 \mathrm{~mm}^{2}$ were placed on different part of the residual normal rectum with the same location in ADC maps. The average values caculated by the two ROIs were regarded as $\mathrm{SI}_{\text {rectum. }}$.

3. One ROI of $100 \mathrm{~mm}^{2}$ was placed on the right gluteus maximus with the same location in ADC maps. The SD was regarded as the background noise: $\mathrm{SD}_{\text {background-DWI }}$ for DW images.

Signal-noise ratio (SNR) and contrast-to-noise ratio (CNR), defined below, were calculated for each DW images.

$$
\begin{aligned}
& \mathrm{SNR}=\mathrm{SI}_{\text {lesion }} / \mathrm{SD}_{\text {background-DWI }} \\
& \mathrm{CNR}=\left(\mathrm{SI}_{\text {lesion }}-\mathrm{SI}_{\text {rectum }}\right) / \mathrm{SD}_{\text {background-DWI }}
\end{aligned}
$$

\section{Pathological grouping for primary rectal cancer}

For the primary rectal cancer, 2 pathological groups were be divided according to their surgical pathological results in cohorts of iShim-DWI and SS-EPI-DWI, respectively:

Group 1 (rectal cancer with relatively well differentiation): patients with carcinoma in situ and rectal cancer of Grade 1 (high differentiated in pathology).

Group 2 (rectal cancer with relatively poor differentiation): patients with Grade 2 (moderate differentiated in pathology) and Grade 3 (poor differentiated in pathology).

\section{Statistical analysis}

SPSS software (version 24.0; SPSS, Chicago, Ill) was used for the statistical analysis. Firstly, nonparametric analysis (Mann-Whitney U tests) were applied to assess fat suppression, artifacts and image quality and to compare iShim- and SS-EPI-DWI, ADC maps. Secondly, Independent- $t$-test analysis was applied to assess quantitative $\mathrm{SD}_{\text {background }}$ (both DWI and ADC maps), SNR and CNR of iShim- and SS-EPI-DWI.

For primary rectal tumor, paired-t-test analysis was applied between lesions and normal rectums for both $\left(\mathrm{SI}_{\text {lesion }}-\mathrm{SI}_{\text {rectum }}\right)$ and $\left(\mathrm{ADC} \mathrm{C}_{\text {lesion }}-\mathrm{ADC}_{\text {rectum }}\right)$ to explore if SI or ADC value of iShim could distinguish lesions from normal rectum. The analysis was conducted in cohort of iShim-DWI and SS-EPI-DWI, respectively.

For primary rectal cancer, independent- $t$-test analysis was applied for $\mathrm{SI}_{\text {lesion }}$ of DWI, $\mathrm{ADC}_{\text {lesion }}$ between two pathologic groups in cohort of iShim-DWI and SS-EPIDWI, respectively. Finally, receiver operator curve (ROC) analysis was performed in $\mathrm{SI}_{\text {lesion }}$ and $\mathrm{ADC}_{\text {lesion }}$

\begin{tabular}{|c|c|c|c|c|c|c|c|c|c|c|c|}
\hline \multirow[b]{2}{*}{ Cohort } & & & \multicolumn{3}{|c|}{ Fat suppression } & \multicolumn{3}{|l|}{ Artifacts } & \multicolumn{3}{|c|}{ Imaging quality } \\
\hline & & & Scores & $Z$ & $P$ & Scores & $Z$ & $P$ & Scores & $Z$ & $P$ \\
\hline \multirow[t]{5}{*}{ Total } & DWI & b1000 & $3.25 \pm 0.51$ & - & & $2.87 \pm 0.45$ & - & & $4.35 \pm 0.53$ & - & \\
\hline & & b800 & $3.92 \pm 0.27$ & -9.078 & $<0.001^{*}$ & $2.94 \pm 0.24$ & -0.804 & 0.422 & $4.82 \pm 0.38$ & -6.439 & $<0.001^{*}$ \\
\hline & & b1600 & $3.94 \pm 0.24$ & -9.402 & $<0.001^{*}$ & $2.92 \pm 0.27$ & -0.281 & 0.779 & $4.88 \pm 0.33$ & -7.381 & $<0.001^{*}$ \\
\hline & ADC Map & SS-EPI & - & & & $2.91 \pm 0.28$ & -1.034 & 0.301 & $4.3 \pm 0.46$ & -6.946 & $<0.001^{*}$ \\
\hline & & iShim & & & & $2.95 \pm 0.21$ & & & $4.8 \pm 0.4$ & & \\
\hline \multirow{5}{*}{$\begin{array}{l}\text { Primary } \\
\text { rectal tumor }\end{array}$} & DWI & b1000 & $3.34 \pm 0.51$ & - & & $2.89 \pm 0.4$ & - & & $4.42 \pm 0.56$ & - & \\
\hline & & b800 & $3.97 \pm 0.16$ & -7.75 & $<0.001^{*}$ & $2.96 \pm 0.2$ & -9.27 & 0.354 & $4.9 \pm 0.3$ & -5.796 & $<0.001^{*}$ \\
\hline & & b1600 & $3.97 \pm 0.16$ & -7.75 & $<0.001^{*}$ & $2.93 \pm 0.25$ & -0.237 & 0.812 & $4.93 \pm 0.25$ & -6.214 & $<0.001^{*}$ \\
\hline & ADC Map & SS-EPI & - & & & $2.92 \pm 0.27$ & -0.896 & 0.37 & $4.34 \pm 0.48$ & -6.49 & $<0.001^{*}$ \\
\hline & & iShim & & & & $2.96 \pm 0.2$ & & & $4.88 \pm 0.33$ & & \\
\hline \multirow[t]{5}{*}{ After treatment } & DWI & b1000 & $3.04 \pm 0.43$ & - & & $2.81 \pm 0.56$ & - & & $4.19 \pm 0.4$ & - & \\
\hline & & b800 & $3.79 \pm 0.42$ & -4.994 & $<0.001^{*}$ & $2.89 \pm 0.31$ & -0.14 & 0.889 & $4.61 \pm 0.5$ & -3.164 & $0.002^{*}$ \\
\hline & & b1600 & $3.86 \pm 0.36$ & -5.465 & $<0.001^{*}$ & $2.89 \pm 0.31$ & -0.14 & 0.889 & $4.75 \pm 0.44$ & -4.156 & $<0.001^{*}$ \\
\hline & ADC Map & SS-EPI & - & & & $2.89 \pm 0.32$ & -0.507 & 0.612 & $4.22 \pm 0.42$ & -2.867 & $0.004^{*}$ \\
\hline & & iShim & & & & $2.93 \pm 0.26$ & & & $4.61 \pm 0.5$ & & \\
\hline
\end{tabular}

Table 1 Results of Qualitative Image quality comparison between iShim- and SS-EPI-DWI

$P$ with ${ }^{*}$ suggested statistical significance. All the $\mathrm{t}$ and $P$ values are the comparison between $\mathrm{b}=800 \mathrm{~s} / \mathrm{mm}^{2}$ of iShim-DWI and $\mathrm{b}=1000 \mathrm{~s} / \mathrm{mm}{ }^{2}$ of SS-EPI-DWI, $\mathrm{b}=1600 \mathrm{~s} / \mathrm{mm}^{2}$ of iShim-DWI and $\mathrm{b}=1000 \mathrm{~s} / \mathrm{mm}^{2}$ of SS-EPI-DWI for each DWI item 
between the two groups to explore if DWI (both iShimand SS-EPI-DWI) could help differentiate them. $P<0.05$ was considered indicative of a significant difference.

\section{Results}

\section{Results of feasibility experiment}

In the 2 patients performed with both iShim- and SSEPI-DWI, higher scores regarding adequate fat suppression and image quality were obtained in iShimDWI (both images of b800 and b1600). Scores of 3.5, 4 and 4 were obtained in fat supprestion for images of $\mathrm{b}=1000,800$ and $1600 \mathrm{~s} / \mathrm{mm}^{2}$, respectively. Scores of $4.5,5$ and 5 were obtained in image quality for images of $\mathrm{b}=1000,800$ and $1600 \mathrm{~s} / \mathrm{mm}^{2}$, respectively. In addition, average SNR of lesions were $21.5,38.4$ and 30.2 for images of $b=1000,800$ and $1600 \mathrm{~s} / \mathrm{mm}^{2}$, respectively. Average CNR of lesions were 10.6, 16.8 and 15.9 for images of $b=1000,800$ and $1600 \mathrm{~s} / \mathrm{mm}^{2}$, respectively.

For ADC maps, higher scores regarding image quality were also obtained in iShim-DWI compared with SSEPI-DWI. Scores of 4.5, 5 and 5 were obtained in image quality for images of $b=1000,800$ and $1600 \mathrm{~s} / \mathrm{mm}^{2}$, respectively.

\section{Patients and clinical information}

Among 101 patients underwent iShim-DWI, 63 were males and 38 were females, with the mean age of $62.1 \pm$ 11.4 years (32 to 83 years); 73 patients were primary rectal tumor while 28 patients were post-surgery or CRT.
Among the 92 patients underwent SS-EPI-DWI, 64 were males and 28 were females, with the mean age of $60.9 \pm 12.6$ years ( 24 to 93 years); 65 patients were primary rectal tumor while the rest 27 patients were postsurgery or CRT.

\section{Pathological results for primary rectal tumor of iShim- DWI}

Pathological results were obtained for all the primary rectal tumor in both cohorts of iShim-DWI and SS-EPIDWI.

In patients of iShim-DWI, 7 patients were confirmed as non-adenocarcinoma ( 1 for solitary fibroma, 2 for stromal tumor, 2 for benign adenoma, and 2 for neuroendocrine tumor). The rest 66 patients were confirmed as primary rectal adenocarcinoma (Carcinoma in situ $(n=8)$, Grade $1(n=3)$, Grade $2(n=47)$, Grade $3(n=$ 8)) for the pathological grouping. Among them, 11 patients were included in Group 1 and 55 patients were included in Group 2.

In patients of SS-EPI-DWI, 2 patients were confirmed as non-adenocarcinoma ( 1 for malignant melanoma, 1 for neuroendocrine tumor), 3 patients were confirmed as mucinous adenocarcinoma. The rest 60 patients were confirmed as primary rectal cancer (Carcinoma in situ $(n=1)$, Grade $1(n=4)$, Grade $2(n=52)$, Grade $3(n=$ 3)) for the pathological grouping. Among them, 5 patients were included in Group 1 and 55 patients were included in Group 2.

Table 2 Results of Quantitative Image quality comparison between iShim- and SS-EPI-DWI

\begin{tabular}{|c|c|c|c|c|c|c|c|c|c|c|c|}
\hline & & & $S D_{\text {background }}$ & & & SNR & & & CNR & & \\
\hline Cohort & & & Values & $t$ & $P$ & Ratio & t & $P$ & Ratio & $t$ & $P$ \\
\hline \multirow[t]{5}{*}{ Total } & DWI & b1000 & $5.99 \pm 1.59$ & - & & - & & & - & & \\
\hline & & b800 & $6.34 \pm 2.49$ & 1.144 & 0.254 & & & & & & \\
\hline & & b1600 & $4.53 \pm 1.51$ & -6.54 & $<0.001^{*}$ & & & & & & \\
\hline & ADC Map & SS-EPI & $174.68 \pm 116.46$ & -7.174 & $<0.001^{*}$ & & & & & & \\
\hline & & iShim & $88.3 \pm 31.43$ & & & & & & & & \\
\hline \multirow{5}{*}{$\begin{array}{l}\text { Primary } \\
\text { rectal } \\
\text { tumor }\end{array}$} & DWI & b1000 & $5.96 \pm 1.56$ & - & & $22.57 \pm 8.66$ & - & & $10.81 \pm 5.83$ & - & \\
\hline & & b800 & $6.11 \pm 2.44$ & 0.436 & 0.063 & $41.26 \pm 17.02$ & 8.157 & $<0.001^{*}$ & $21.53 \pm 11.46$ & 6.948 & $<0.001^{*}$ \\
\hline & & b1600 & $4.49 \pm 1.54$ & -5.542 & $<0.001^{*}$ & $35.35 \pm 14.76$ & 6.208 & $<0.001^{*}$ & $22.59 \pm 11.95$ & 7.39 & $<0.001^{*}$ \\
\hline & ADC Map & SS-EPI & $179.71 \pm 134.98$ & -5.46 & $<0.001^{*}$ & - & & & - & & \\
\hline & & iShim & $91.2 \pm 29.51$ & & & & & & & & \\
\hline \multirow[t]{5}{*}{ After treatment } & DWl & b1000 & $6.08 \pm 1.71$ & - & & - & & & - & & \\
\hline & & b800 & $6.93 \pm 2.57$ & 1.432 & 0.158 & & & & & & \\
\hline & & b1600 & $4.64 \pm 1.43$ & -3.399 & $0.001^{*}$ & & & & & & \\
\hline & ADC Map & SS-EPI & $162.59 \pm 49.08$ & -7.108 & $<0.001^{*}$ & & & & & & \\
\hline & & iShim & $80.77 \pm 35.44$ & & & & & & & & \\
\hline
\end{tabular}

$P$ with ${ }^{*}$ suggested statistical significance. All the $\mathrm{t}$ and $P$ values are the comparison between $\mathrm{b}=800 \mathrm{~s} / \mathrm{mm}^{2}$ of iShim-DWI and $\mathrm{b}=1000 \mathrm{~s} / \mathrm{mm}{ }^{2}$ of SS-EPI-DWI, $\mathrm{b}=1600 \mathrm{~s} / \mathrm{mm}^{2}$ of iShim-DWI and $\mathrm{b}=1000 \mathrm{~s} / \mathrm{mm}^{2}$ of SS-EPI-DWI for each DWI item 


\section{Qualitative assessment}

For DW images comparison, significantly higher scores regarding adequate fat suppression and image quality were obtained in patients using iShim-DWI (both images of $b=800$ and $1600 \mathrm{~s} / \mathrm{mm}^{2}$ ) in all the cohorts (total cohort, cohort of primary rectal tumor and cohort of after treatment) by Mann-Whitney $U$ tests. No significant differences were obtained for the comparison of artifacts between iShim- and SS-EPIDWI (Table 1).

For ADC maps, significantly higher scores regarding image quality were obtained in patients using iShimDWI in all the cohorts. And significantly higher score regarding artifacts was obtained in the cohort of primary rectal tumor using iShim-DWI by Mann-Whitney U tests (Table 1).

\section{Quantitative assessment}

$\mathrm{SD}_{\text {background }}$ showed significantly decreasing in $\mathrm{b}=1600$ $\mathrm{s} / \mathrm{mm}^{2}$ images and ADC maps of iShim-DWI in all the cohorts, compared with SS-EPI-DWI by paired-t-test analyses (Table 2).

Both SNR and CNR of $\mathrm{b}=800$ and $1600 \mathrm{~s} / \mathrm{mm}^{2} \mathrm{im}-$ ages in iShim-DWI were significantly higher than those of $b=1000 \mathrm{~s} / \mathrm{mm}^{2}$ images in SS-EPI-DWI. Meanwhile, $b=800 \mathrm{~s} / \mathrm{mm}^{2}$ images showed the highest SNR among the 3 sets of DW images while $b=1600$ $\mathrm{s} / \mathrm{mm}^{2}$ images obtained the highest CNR (Table 2, Figs. 2, 3 and 4).

-In 66 patients of primary rectal cancer of iShim-DWI, paired-t-test showed that $\mathrm{SI}_{\text {lesion }}$ was significantly higher than $\mathrm{SI}_{\text {rectum }}$ in both $\mathrm{b}=800$ and $1600 \mathrm{~s} / \mathrm{mm}^{2}$ images. In addition, $\mathrm{ADC}_{\text {lesion }}$ was also significantly lower than

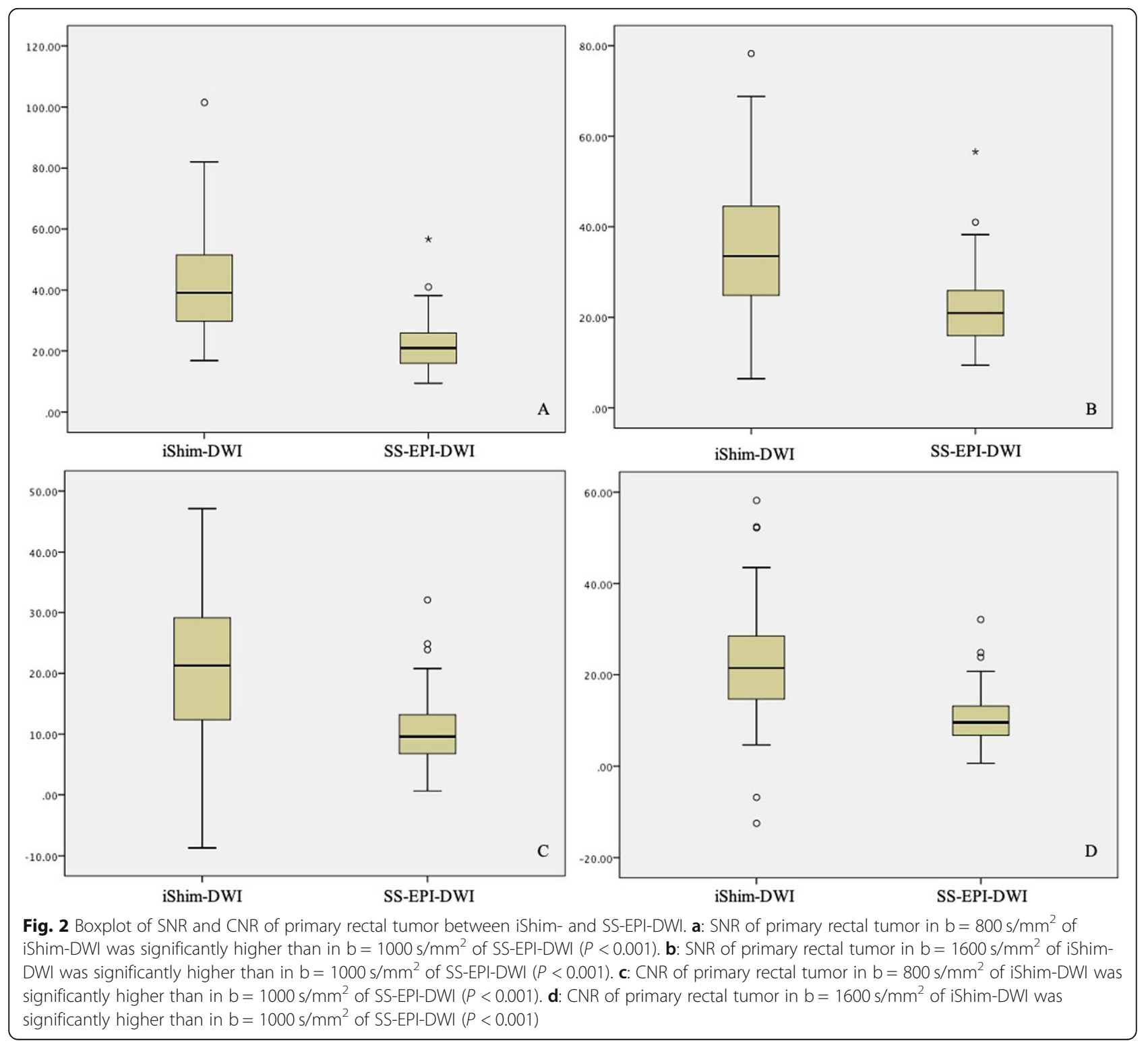




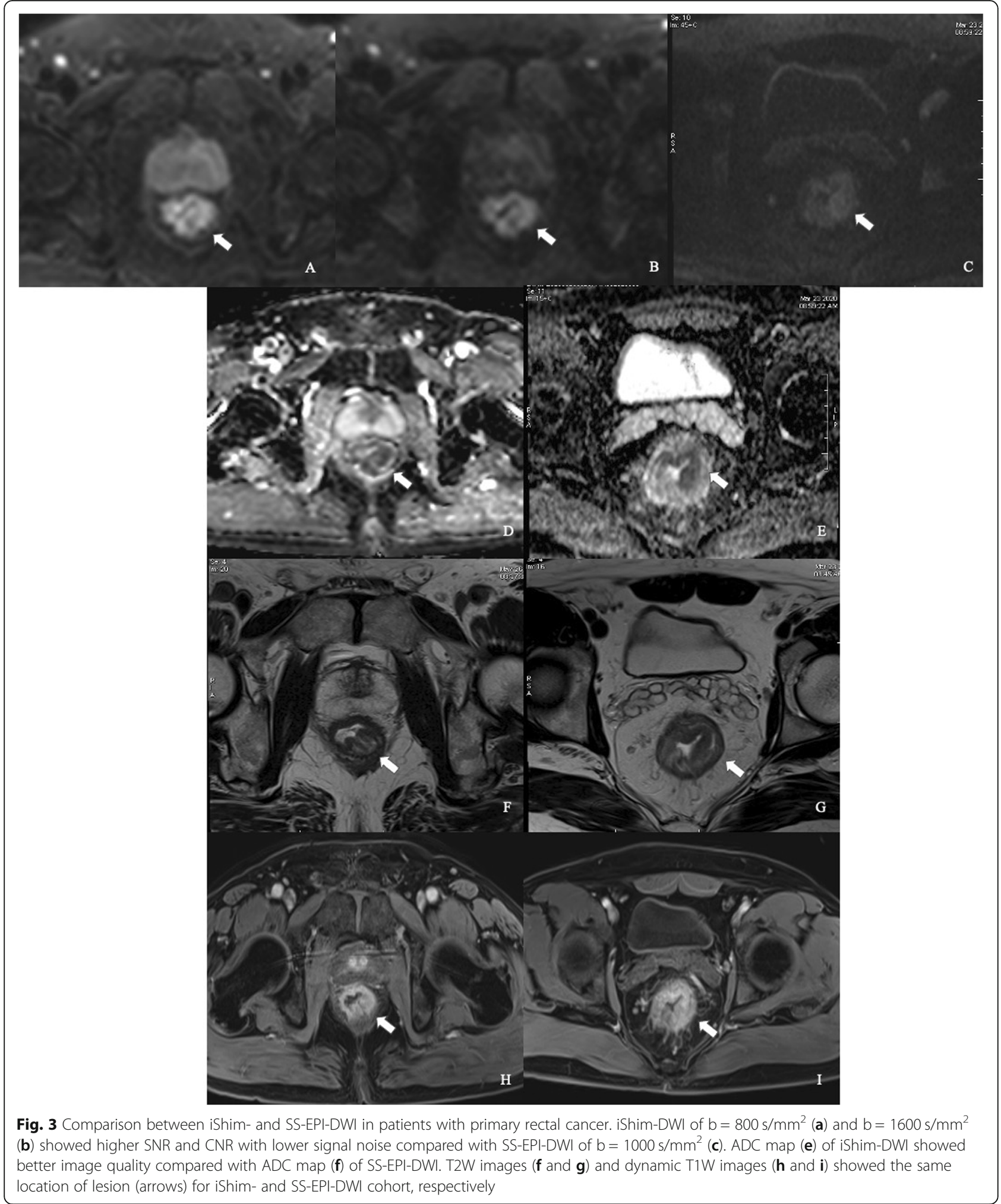

$\mathrm{ADC}_{\text {rectum. }}$ Between the two pathologic groups, $\mathrm{ADC}$ values was significantly lower in Group 2 (mean value: $\left.(0.733 \pm 0.08) \times 10^{-3} \mathrm{~mm}^{2} / \mathrm{s}\right)$ than in Group 1 (mean value: $\left.(0.912 \pm 0.21) \times 10^{-3} \mathrm{~mm}^{2} / \mathrm{s}\right)$. There was no significant difference between $\mathrm{SI}_{\text {lesion }}$ of $\mathrm{b}=800 \mathrm{~s} / \mathrm{mm}^{2}$ images, but $\mathrm{SI}_{\text {lesion }}$ of $\mathrm{b}=1600 \mathrm{~s} / \mathrm{mm}^{2}$ images showed 


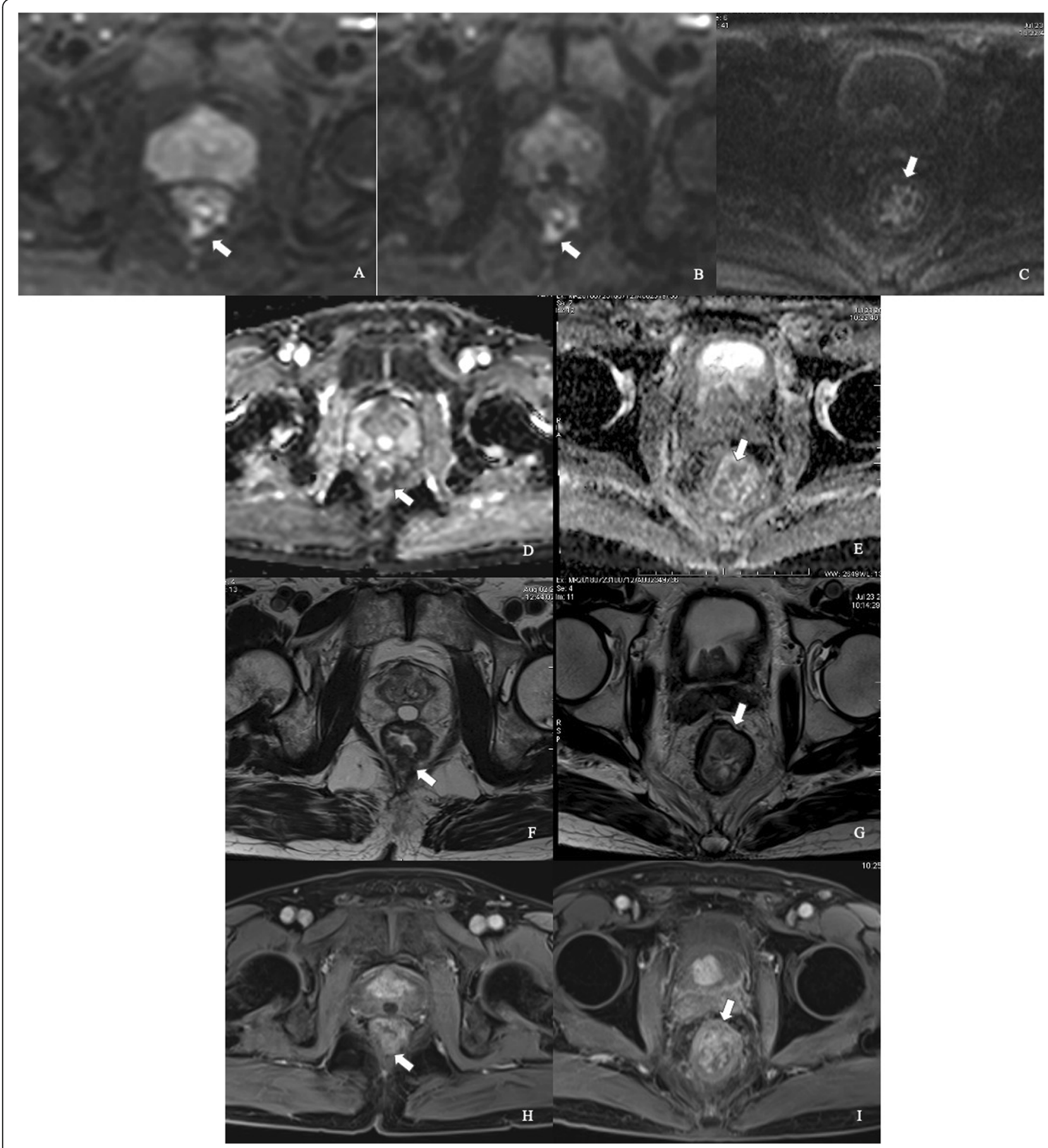

Fig. 4 Comparison between iShim- and SS-EPI-DWI in patients after CRT. iShim-DWI of $b=800 \mathrm{~s} / \mathrm{mm}^{2}$ (a) and b=1600 s/mm² (b) showed higher SNR and CNR with lower signal noise compared with SS-EPI-DWI of $b=1000 \mathrm{~s} / \mathrm{mm}^{2}$ (c). ADC map (e) of iShim-DWI showed better image quality compared with ADC map (f) of SS-EPI-DWI. T2W images ( $\mathbf{f}$ and $\mathbf{g}$ ) and dynamic T1W images (h and $\mathbf{i})$ showed the same location of lesion (arrows) for iShim- and SS-EPI-DWI cohort, respectively

significantly different between the two groups. ROC analyses showed significance of $\mathrm{ADC}$ values and $\mathrm{SI}_{\text {lesion }}$ between the two groups (Table 3, Figs. 5, 6 and 7).

-In 60 patients of primary rectal cancer of SS-EPIDWI, paired-t-test showed that $\mathrm{SI}_{\text {lesion }}$ was significantly higher than $\mathrm{SI}_{\text {rectum }}$ in $\mathrm{b}=1000 \mathrm{~s} / \mathrm{mm}^{2}$ images. In addition, $\mathrm{ADC}_{\text {lesion }}$ was also significantly lower than $\mathrm{ADC}_{\text {rectum }}$ (Table 3). In two pathologic groups of SS-EPI-DWI, there was no significant difference between them in both SI of DWI and in 
Table 3 Results of Quantitative Assessment of primary rectal cancer

\begin{tabular}{|c|c|c|c|c|c|c|c|}
\hline & & & $t$ test & & & ROC analysis & \\
\hline & Images & & Average & $\mathrm{t}$ & $P$ & Area under curve & $P$ \\
\hline \multirow[t]{2}{*}{ Sl lesion } & \multirow[t]{2}{*}{ b800 } & Sl lesion & $221.68 \pm 43.51$ & 16.868 & $<0.001^{*}$ & - & \\
\hline & & $\mathrm{SI}_{\text {rectum }}$ & $103.99 \pm 42.68$ & & & & \\
\hline \multirow[t]{4}{*}{$\mathrm{SI}_{\text {rectum }}$} & \multirow[t]{2}{*}{ b1600 } & Sl lesion & $143.28 \pm 32.9$ & 20.468 & $<0.001^{*}$ & & \\
\hline & & $\mathrm{SI}_{\text {rectum }}$ & $48.94 \pm 23.69$ & & & & \\
\hline & \multirow[t]{2}{*}{ b1000 } & Sl lesion & $125.86 \pm 32.14$ & 17.441 & $<0.001^{*}$ & & \\
\hline & & $\mathrm{SI}_{\text {rectum }}$ & $65.51 \pm 15.57$ & & & & \\
\hline \multirow[t]{2}{*}{$A D C_{\text {lesion }}$} & \multirow[t]{2}{*}{ iShim-DWI } & $A D C_{\text {lesion }}$ & $0.763 \pm 0.13$ & -11.103 & $<0.001^{*}$ & & \\
\hline & & $A D C_{\text {rectum }}$ & $1.294 \pm 0.36$ & & & & \\
\hline \multirow{2}{*}{$A D C_{\text {rectum }}$} & \multirow[t]{2}{*}{ SS-EPI-DWI } & $A D C_{\text {lesion }}$ & $0.768 \pm 0.13$ & -17.303 & $<0.001^{*}$ & & \\
\hline & & $A D C_{\text {rectum }}$ & $1.243 \pm 0.22$ & & & & \\
\hline \multirow{6}{*}{$\begin{array}{l}\text { Group } \\
\text { Comparison } \\
\text { In iShim-DWI }\end{array}$} & \multirow{2}{*}{$\begin{array}{l}\text { ADC } \text { Iesion } \\
\left(\times 10^{-3} \mathrm{~mm}^{2} / \mathrm{s}\right)\end{array}$} & Group 1 & $0.912 \pm 0.21$ & 2.837 & $0.017^{*}$ & 0.742 & $0.012^{*}$ \\
\hline & & Group 2 & $0.732 \pm 0.084$ & & & & \\
\hline & \multirow{2}{*}{$\begin{array}{l}\text { Sl lesion } \\
\text { of b800 }\end{array}$} & Group 1 & $191.91 \pm 68.08$ & -1.697 & 0.118 & 0.692 & $0.046^{*}$ \\
\hline & & Group 2 & $227.64 \pm 34.71$ & & & & \\
\hline & \multirow{2}{*}{$\begin{array}{l}\text { Sl lesion } \\
\text { of b1600 }\end{array}$} & Group 1 & $113.95 \pm 47.67$ & -2.379 & $0.036^{*}$ & 0.756 & $0.008^{*}$ \\
\hline & & Group 2 & $149.15 \pm 25.92$ & & & & \\
\hline \multirow{4}{*}{$\begin{array}{l}\text { Group } \\
\text { Comparison } \\
\text { In SS-EPI-DWI }\end{array}$} & \multirow{2}{*}{$\begin{array}{l}\mathrm{ADC}_{\text {lesion }} \\
\left(\left(\times 10^{-3} \mathrm{~mm}^{2} / \mathrm{s}\right)\right.\end{array}$} & Group 1 & $0.815 \pm 0.13$ & 1.029 & 0.308 & 0.619 & 0.382 \\
\hline & & Group 2 & $0.764 \pm 0.1$ & & & & \\
\hline & \multirow{2}{*}{$\begin{array}{l}\text { Sl lesion } \\
\text { of } b=1000\end{array}$} & Group 1 & $125.1 \pm 29.69$ & 0.663 & 0.51 & 0.55 & 0.714 \\
\hline & & Group 2 & $134.6 \pm 40.71$ & & & & \\
\hline
\end{tabular}

$P$ with * suggested statistical significance

ADC values. ROC analyses also showed no significance in both $\mathrm{SI}_{\text {lesion }}$ and $\mathrm{ADC}$ values between the two groups (Table 3).

\section{Discussion}

In this study, we investigated the qualitative and quantitative performance of iShim-DWI in rectal cancer imaging, compared with SS-EPI-DWI. The results demonstrated that iShim-DWI significantly outperformed SS-EPI-DWI in SNR and CNR of primary rectal tumor. Both SI and ADC values from iShim-DWI showed significant difference between primary rectal tumor and residual normal rectum. In addition, SI from $\mathrm{b}=1600 \mathrm{~s} / \mathrm{mm}^{2}$ images and ADC values have potential value in characterize well-differentiated and poordifferentiated rectal cancer.

DW image quality plays an important role in detection and characterization of lesions. Previous studies [16-22] have conducted research on improving image quality of DWI techniques such as iShim, readout segmentation of long variable echo trains (RESOLVE), multiplexed sensitivity-encoding (MUSE). Owing to the procedure of dynamic frequency adjustment and slice-selective shimming that reduces field inhomogeneities and thus related artifacts [18-20, 23], iShim-DWI showed superior performance in lesion detection of bladder, breast, neck and so on. Our study also demonstrated it could improve image quality in rectal tumor compared to SSEPI-DWI and lesion detection with higher SNR and CNR. With the acceptable scanning time (less than 3 min), iShim-DWI has great potential application in routine work.

A few studies explored DWI with an ultra-high bvalue (more than $1000 \mathrm{~s} / \mathrm{mm}^{2}$ ) and showed it might be a promising technique for detecting malignant tumors [911]. For example, Bittencourt et al. found that DWI with b-value of $1400 \mathrm{~s} / \mathrm{mm}^{2}$ showed benefits in increasing rates of detection of prostate cancer [9]. Also in our previous study of rectal cancer [10], we indicated that bvalue of $2000 \mathrm{~s} / \mathrm{mm}^{2}$ could be helpful in detecting lesions with a clear demarcation of borders. DWI with ultra-high b value could be helpful to obtain a nearly perfect background suppression (less 'T2-shinethrough'), but the image quality of $b=2000 \mathrm{~s} / \mathrm{mm}^{2}$ did not showed any superiority when compared with that of $\mathrm{b}=800 \mathrm{~s} / \mathrm{mm}^{2}$, and even achieved lower scores in patients after CRT. However, DWI of $b=1600 \mathrm{~s} / \mathrm{mm}^{2}$ achieved best image qualities and highest CNR compared to those of $b=800$ and $1000 \mathrm{~s} / \mathrm{mm}^{2}$ in both patients with primary rectal tumor and after treatment. 


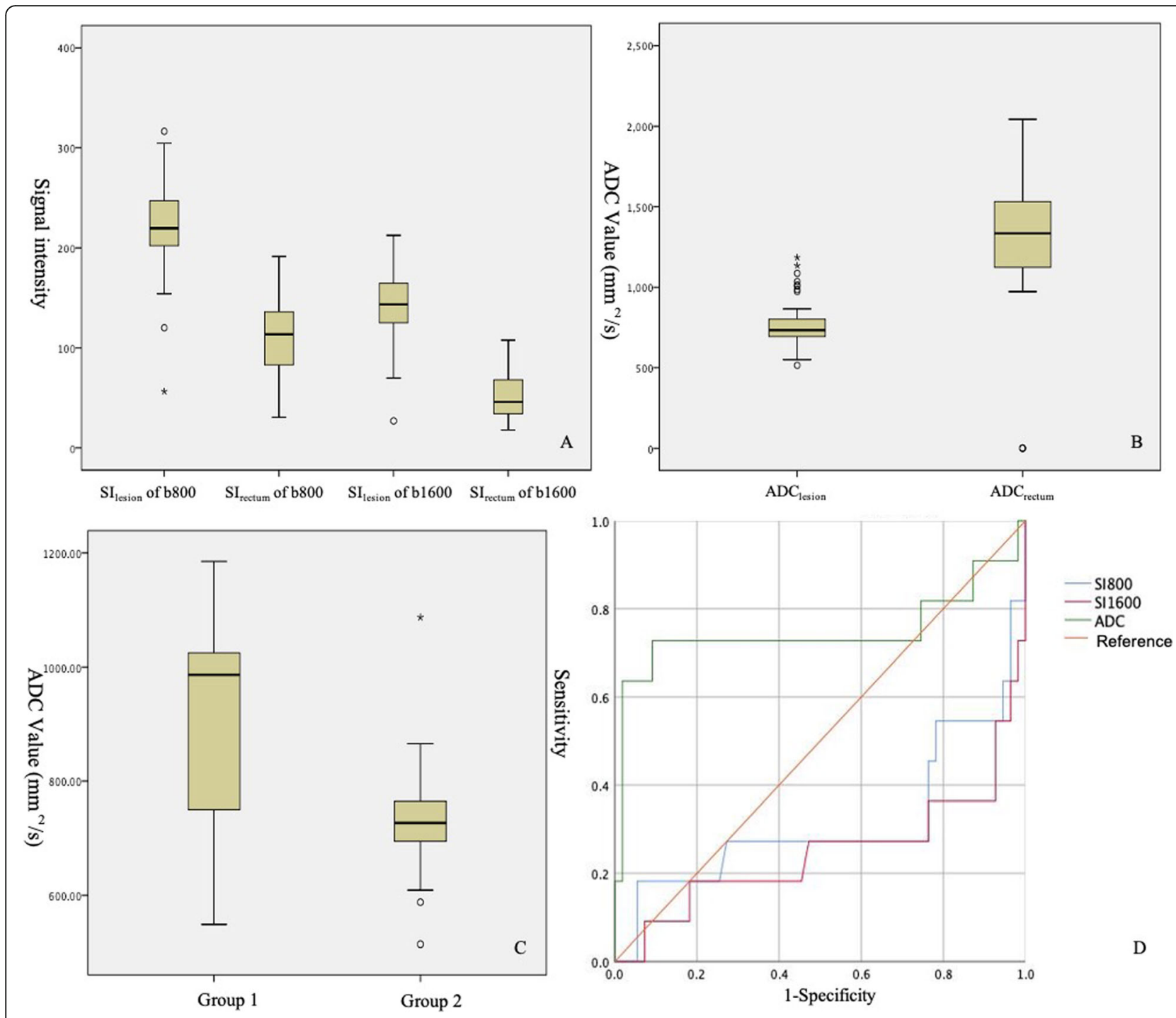

Fig. 5 Boxplot of Quantitative Assessment of primary rectal cancer in iShim-DWI. a: Signal intensity (SI) showed significantly high in primary rectal cancer compared with residual rectal wall in iShim-DWI. b: ADC values shoed significantly low in primary rectal cancer compared with residual rectal wall in iShim-DWI. c: ADC values comparison showed significant difference between two pathological groups (Group 1: well differentiated cancer and Group 2: moderate to lower differentiated cancer). $\mathbf{d}$ : ROC analysis between two pathological groups showed statistical significance in Sl lesion of $b=800 \mathrm{~s} / \mathrm{mm}^{2}(\mathrm{AUC}=0.692, P=0.046)$, Sl lesion of $\mathrm{b}=1600 \mathrm{~s} / \mathrm{mm}^{2}(\mathrm{AUC}=0.756, P=0.008)$ and $\mathrm{ADC}(\mathrm{AUC}=0.742, P=0.012)$ maps, respectively

Therefore, DWI of $\mathrm{b}=1600 \mathrm{~s} / \mathrm{mm}^{2}$ might be a better choice for ultra-high $b$ value in rectal cancer in future study.

Another important reason for this result might be attributed to different DWI techniques used: iShim used in our study and SS-EPI in previous studies [9-11]. The surrounding tissue around rectum may induce inhomogeneity of DWI signal, such as bones, bowel movement and bowel contents. The advantage of iShim-DWI was to reduce the inhomogeneity and improve image quality. From our results, image quality showed higher in $\mathrm{b}=$ $800 \mathrm{~s} / \mathrm{mm}^{2}$ of iShim-DWI than $\mathrm{b}=1000 \mathrm{~s} / \mathrm{mm}^{2}$ of SSEPI-DWI. We speculated that iShim-DWI itself worth application in rectal tumor and will improve the lesion detection greatly.

ADC value, which is derived from DWI, is an important quantitative biomarker revealing lesion characterization. Previous studies have already investigated the use of SSEPI-DWI as a tool for treatment response prediction and have shown that low pretreatment ADC values may correlate with a good response to CRT [24-27]. Another study also showed that an early increase of mean ADC value in rectal cancer correlates with a good response to CRT [28]. However, most studies focused on the investigation of ADC as a biomarker to assess or predict response to treatment. Few information of ADC on 


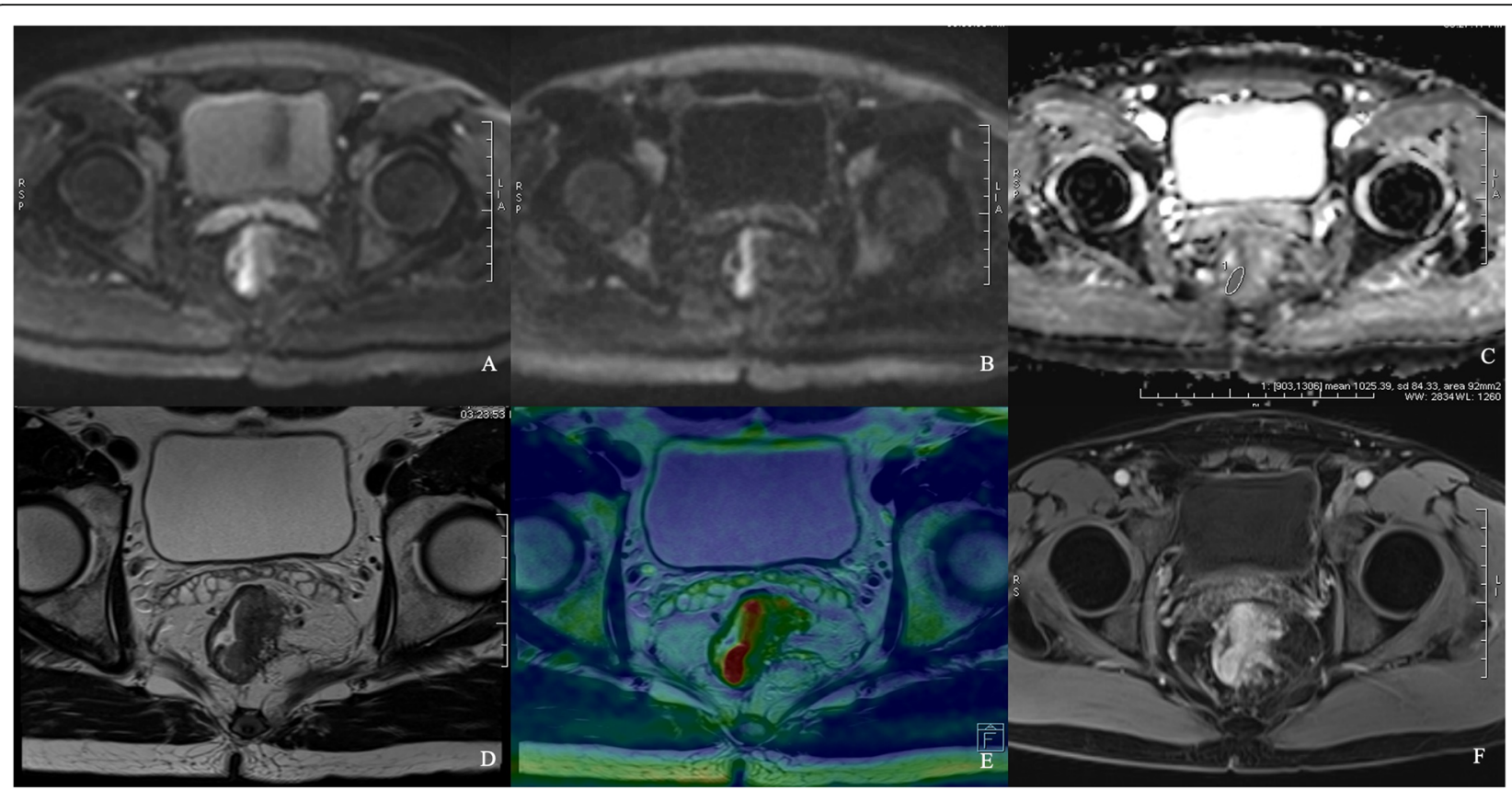

Fig. 6 iShim DWl for patient with primary rectal cancer of Grade 1. DW images of $b=800 \mathrm{~s} / \mathrm{mm}^{2}(\mathbf{a}), b=1600 \mathrm{~s} / \mathrm{mm}^{2}$ (b), ADC map (c), T2Wl (d), infusion images of both T2WI and DWI (e) and dynamic T1WI (f) showed the same location of lesion. Average ADC value measured was $1025.39 \times 10^{-6} \mathrm{~mm}^{2} / \mathrm{s}(\mathbf{c})$

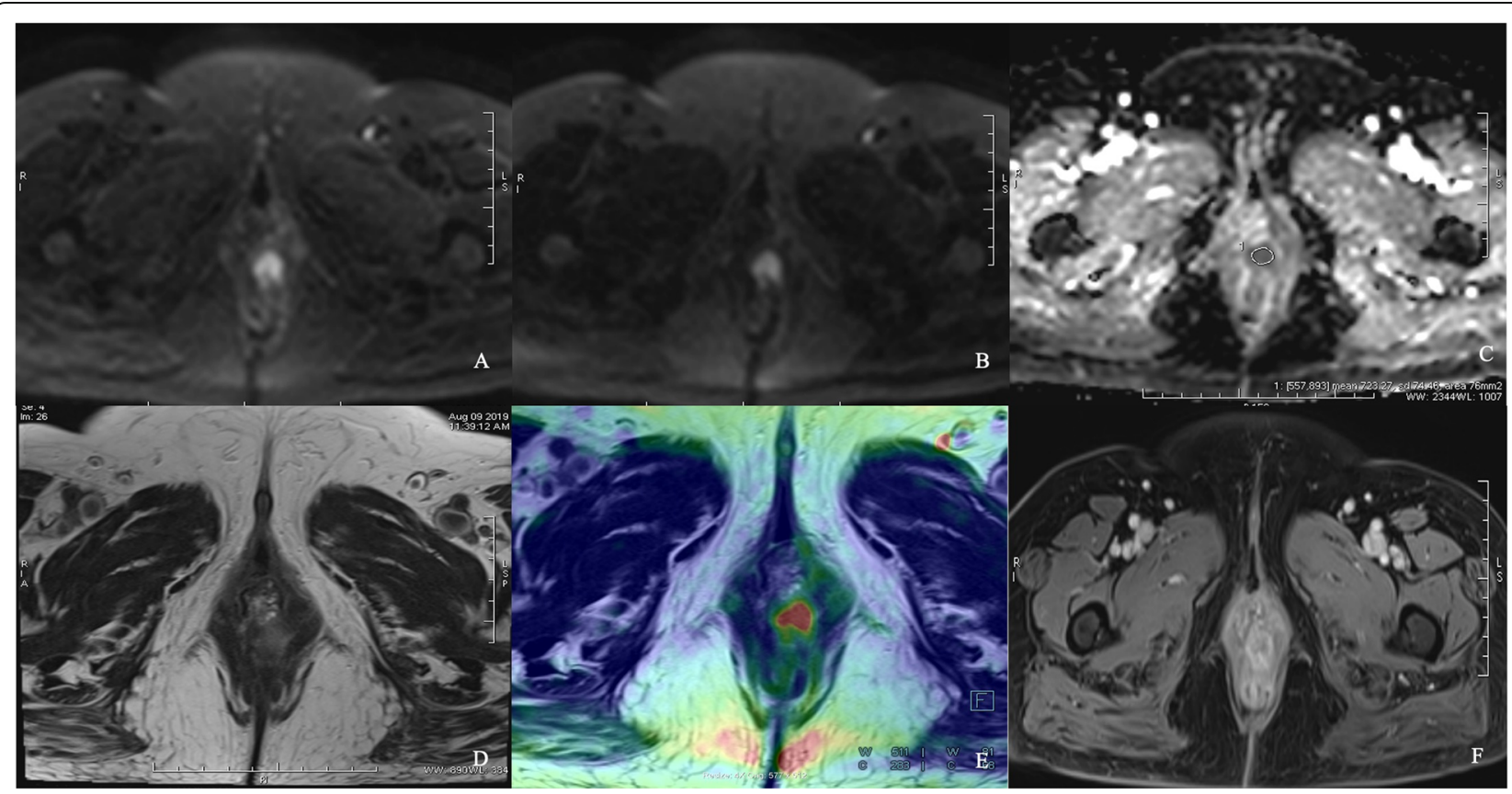

Fig. 7 IShim DWI for patient with primary rectal cancer of Grade 3. DW images of $b=800 \mathrm{~s} / \mathrm{mm}^{2}(\mathbf{a}), b=1600 \mathrm{~s} / \mathrm{mm}^{2}$ (b), ADC map (c), T2WI (d), infusion images of both T2WI and DWI (e) and dynamic T1WI (F) showed the same location of lesion. Average ADC value measured was $723.27 \times 10^{-6} \mathrm{~mm}^{2} / \mathrm{s}(\mathbf{c})$ 
differentiation of primary rectal cancer has been known. And our resuts showed iShim-DWI may have the potential ability. As a non-invasive technique, this ability of iShim-DWI should gained more attention to for predicting pathological results.

However, in our study, ADC value generated by iShim-DWI combination of $b=0,800$ and $1600 \mathrm{~s} /$ $\mathrm{mm}^{2}$ could be helpful in differentiating rectal cancer with well- from poor-differentiation, but SS-EPI-DWI could not. From results of previous experiment, ADC value could be generated differently due to different $\mathrm{b}$ values combination [11]. Although iShim-DWI have improved the image quality with less artifacts, the technique might not be the main contributor to tumor differentiation. The combination of proper high and ultra-high $b$ values should be the reason. More patients with well-differentiated rectal cancer should be enrolled in furture, and also we will continue to explore its treatment prediction in this cohort of patients with follow-ups.

Paired-t-test analysis showed SI of primary rectal cancer (in both $\mathrm{b}=800$ and $1600 \mathrm{~s} / \mathrm{mm}^{2}$ DWI) significantly different from residual normal rectal wall in our study. The benefits for this result might help determine the borderline of tumor more precisely. However, an overlap existed in both SI and ADC value between the two tissue. Moreover, the normal rectal wall was always too thin to be drawn ROI on. Small field of view (FOV) techniques combined with iShim-DWI might help further differentiation in future.

There are several limations in our study. First, our study may have statitical bias, because patients enrolled were received different DWI protocols. Considering the heavy clinical workload in routine work, repeat scanning for every patient will increase the amont of the work. However, before the study, we conducted both SS-EPIDWI and iShim-DWI in 2 patients for feasibility experiment, and our results showed the image quality were higher in both DWI images and ADC maps of iShimDWI compared with SS-EPI-DWI. Consecutive patients were selected with DWI protocols randomly applied. In addition, large sample capacity (nearly 100 patients for each cohort) enrolled in our study also could reduce the bias and make our results persuasive. Second, lesion measurement was conducted on 2-dimentional images which may not be as accurate as measuring the whole tumor in three dimensions. To comprensate for that, we used multiple sites measurement to avoid bias as much as possible. In the future, volumetric measurement could be explored by development of measurement tools. Another limitation was the relatively small capacity of welldifferentiated cancer enrolled. Following study should be further conducted with more participants enrolled.

\section{Conclusion}

In conclusion, iShim-DWI with b values of 800 and $1600 \mathrm{~s} / \mathrm{mm}^{2}$ provides improved spatial resolution and image quality for rectal cancer imaging and characterization.

\begin{abstract}
Abbreviations
DWI: Diffusion-weighted imaging; SS-EPI: Single-shot echo-planar imaging; SI: Signal intensity; CRT: Chemo-radiation therapy; ADC: Apparent diffusion coefficient; iShim: Integrated slice-specific dynamic shimming; T2W TSE: T2weighted Turbo Spin Echo; PACS: Picture archiving and communication systems; ROI: Region of interest; SD: Standard deviation; SNR: Signal-noise ratio; CNR: Contrast-to-noise ratio; ROC: Receiver operator curve; RESOLVE: Readout segmentation of long variable echo trains; MUSE: Multiplexed sensitivity-encoding; FOV: Field of view
\end{abstract}

\section{Supplementary Information}

The online version contains supplementary material available at https://doi. org/10.1186/s40644-021-00403-9.

Additional file 1.

Acknowledgements

We thank Thomas Benker from MR Application Development, Siemens Healthcare GmbH, Erlangen, Germany for iShim-DWI technique support, and we also thank GE healthcare for paper polish.

\section{Authors' contributions}

Jianxing Qiu: Data curation, Writing- editing, Visualization, Investigation. Jing Liu1: Data curation, Writing- Original draft preparation. Zhongxu Bi: Visualization, Investigation. Xiaowei Sun: Visualization, Investigation. Xin Wang: Conceptualization, Methodology. Junling Zhang: Conceptualization, Methodology. Chengwen Liu: Software, Validation. Jinxia Zhu: Software, Validation. Naishan Qin: Supervision, Writing- Reviewing and Editing. All authors read and approved the final manuscript.

Funding

Not applicable.

Availability of data and materials

Not applicable.

\section{Declarations}

\section{Ethics approval and consent to participate}

This study was approved by institutional review board. The need for written informed consent was waived by the institutional review board due to the retrospective design of the study.

Consent for publication

Not applicable.

Competing interests

Not applicable.

\section{Author details}

${ }^{1}$ Department of Radiology, Peking University First Hospital, 8 XiShiKu Avenue, XiCheng District, Beijing 100034, China. ${ }^{2}$ Department of Gastrointestinal Surgery, Peking University First Hospital, Beijing, China. ${ }^{3}$ MR Collaboration, Siemens Healthcare, Ltd., Beijing, China.

Received: 27 November 2020 Accepted: 26 March 2021

Published online: 07 April 2021

References

1. Beets-Tan RGH, Lambregts DMJ, Maas M, Bipat S, Barbaro B, Curvo-Semedo $L$, et al. Magnetic resonance imaging for clinical management of rectal cancer: updated recommendations from the 2016 European society of 
gastrointestinal and abdominal radiology (ESGAR) consensus meeting. Eur Radiol. 2018;28(4):1465-75. https://doi.org/10.1007/s00330-017-5026-2.

2. Soyer $P$, Lagadec $M$, Sirol M, Dray X, Duchat D, Vignaud A, et al. Freebreathing diffusion-weighted single-shot echoplanar MR imaging using parallel imaging (GRAPPA 2) and high b value for the detection of primary rectal adenocarcinoma. Cancer Imaging. 2010;10:32-9. https://doi.org/10.11 02/1470-7330.2010.0011.

3. Ichikawa T, Erturk SM, Motosugi U, Sou H, lino H, Araki T, et al. High-B-value diffusion-weighted MRI in colorectal cancer. Am J Roentgenol. 2006;187(1): 181-4. https://doi.org/10.2214/AJR.05.1005.

4. Tomizawa M, Shinozaki F, Uchida Y, Uchiyama K, Fugo K, Sunaoshi T, et al. Diffusion-weighted whole-body imaging with background body signal suppression/ T2 image fusion and positron emission tomography/ computed tomography of upper gastrointestinal cancers. Abdom Imaging 2015;40(8):3012-9. https://doi.org/10.1007/s00261-015-0545-2.

5. Z-H L, C-H H, Qian W-X, Cao W-H. Preoperative diffusion-weighted imaging value of rectal cancer: preoperative T staging and correlations with histological T stage. Clin Imaging. 2016;40(3):563-8. https://doi.org/10.1016/j. clinimag.2015.12.006.

6. Partridge SC, Nissan N, Rahbar H, Kitsch AE, Sigmund EE. Diffusion-weighted breast MRI: clinical applications and emerging techniques. J Magn Reson Imaging. 2017:45(2):337-55. https://doi.org/10.1002/jmri.25479.

7. Jezzard P, Balaban RS. Correction for geometric distortion in echo planar images from B0 field variations. Magn Reson Med. 1995;34(1):65-73. https:// doi.org/10.1002/mrm.1910340111.

8. Rosenkrantz AB, Hindman N, Lim RP, Das K, Babb JS, Mussi TC, et al. Diffusion-weighted imaging of the prostate: comparison of b1000 and b2000 image sets for index lesion detection. J Magn Reson Imaging. 2013; 38(3):694-700. https://doi.org/10.1002/jmri.24016.

9. Bittencourt LK, Attenberger UI, Lima D, Strecker R, de Oliveira A, Schoenberg $\mathrm{SO}$, et al. Feasibility study of computed vs measured high bvalue $\left(1400 \mathrm{~s} / \mathrm{mm}^{2}\right)$ diffusion-weighted MR images of the prostate. World J Radiol. 2014;6(6):374-80. https://doi.org/10.4329/wjr.v6.i6.374.

10. Hausmann D, Liu J, Budjan J, Reichert M, Ong M, Meyer M, et al. Image quality assessment of $2 \mathrm{D}$ versus $3 \mathrm{D} \mathrm{T} 2 \mathrm{WI}$ and evaluation of ultra-high $\mathrm{b}$ value $\left(b=2,000 \mathrm{~mm} / \mathrm{s}^{2}\right)$ DWI for response assessment in rectal cancer. Anticancer Res. 2018;38(2):969-78. https://doi.org/10.21873/anticanres.12311.

11. Chen L, Shen F, Li Z, Lu H, Chen Y, Wang Z, et al. Diffusion-weighted imaging of rectal cancer on repeatability and cancer characterization: an effect of b-value distribution study. Cancer Imaging. 2018;18(1):43. https:// doi.org/10.1186/s40644-018-0177-1.

12. Hu YC, Yan LF, Sun Q, Liu ZC, Wang SM, Han Y, et al. Comparison between ultra-high and conventional mono b-value DWI for preoperative glioma grading. Oncotarget. 2017;8(23):37884-95. https://doi.org/10.18632/oncota rget. 14180 .

13. Bassaneze T, Goncalves JE, Faria JF, Palma RT, Waisberg J. Quantitative aspects of diffusion-weighted magnetic resonance imaging in rectal Cancer response to neoadjuvant therapy. Radiol Oncol. 2017;51(3):270-6. https:// doi.org/10.1515/raon-2017-0025.

14. Intven M, Reerink O, Philippens ME. Repeatability of diffusion-weighted imaging in rectal cancer. J Magn Reson Imaging. 2014;40(1):146-50. https:// doi.org/10.1002/jmri.24337.

15. Pham T, Liney G, Wong K, Rai R, Lee M, Moses D, et al. Study protocol: multi-parametric magnetic resonance imaging for therapeutic response prediction in rectal cancer. BMC Cancer. 2017;17(1):465. https://doi.org/10.11 86/s12885-017-3449-4

16. Liney GP, Holloway L, Al Harthi TM, Sidhom M, Moses D, Juresic E, et al. Quantitative evaluation of diffusion-weighted imaging techniques for the purposes of radiotherapy planning in the prostate. Brit J Radiol. 2015; 88(1049):20150034. https://doi.org/10.1259/bjr.20150034.

17. Chen L, Sun P, Hao Q, Yin W, Xu B, Ma C, et al. Diffusion-weighted MRI in the evaluation of the thyroid nodule: Comparison between integratedshimming EPI and conventional 3D-shimming EPI techniques. Oncotarget. 2018;9(40):26209-16. https://doi.org/10.18632/oncotarget.25279.

18. Li H, Liu L, Shi Q, Stemmer A, Zeng H, Li Y, et al. Bladder cancer: detection and image quality compared among iShim, RESOLVE, and Ss-EPI diffusionweighted MR imaging with high b value at 3.0 T MRI. Medicine (Baltimore). 2017;96(50):e9292. https://doi.org/10.1097/MD.0000000000009292.

19. Gatidis S, Graf H, Weiß J, Alto Stemmer A, Kiefer B, Nikolaou K, et al. Diffusion-weighted echo planar MR imaging of the neck at $3 \mathrm{~T}$ using integrated shimming: comparison of MR sequence techniques for reducing artifacts caused by magnetic-field inhomogeneities. MAGMA. 2017;30(1):5763. https://doi.org/10.1007/s10334-016-0582-z.

20. Lee SK, Tan ET, Govenkar A, Hancu I. Dynamic slice-dependent shim and center frequency update in 3 T breast diffusion weighted imaging. Magn Reson Med. 2014;71(5):1813-8. https://doi.org/10.1002/mrm.24824.

21. Naranjo ID, Gullo RL, Morris EA, Larowin T, Fung MM, Guidon A, et al. Highspatial-resolution multishot multiplexed sensitivity- encoding diffusionweighted imaging for improved quality of breast images and differentiation of breast lesions: a feasibility study. Radiology. 2020;2(3):e190076. https://doi. org/10.1148/rycan.2020190076.

22. Chen NK, Guidon A, Chang HC, Song AW. A robust multi-shot scan strategy for high-resolution diffusion weighted MRI enabled by multiplexed sensitivity-encoding (MUSE). Neuroimage. 2013;72:41-7. https://doi.org/10.1 016/j.neuroimage.2013.01.038.

23. Zhang H, Xue H, Alto S, Hui L, Kannengiesser S, Berthold K, et al. Integrated shimming improves lesion detection in whole-body diffusion-weighted examinations of patients with plasma disorder at 3 T. Investig Radiol. 2016; 51(5):297-305. https://doi.org/10.1097/RLI.0000000000000238.

24. Intven M, Reerink O, Philippens ME. Diffusion-weighted MRI in locally advanced rectal cancer: pathological response prediction after neoadjuvant radiochemotherapy. Strahlenther Onkol. 2013;189(2):117-22. https://doi. org/10.1007/s00066-012-0270-5.

25. Jung SH, Heo SH, Kim JW, Jeong YY, Shin SS, Soung MG, et al. Predicting response to neoadjuvant chemoradiation therapy in locally advanced rectal cancer: diffusion-weighted 3 tesla MR imaging. J Magn Reson Imaging. 2012;35(1):110-6. https://doi.org/10.1002/jmri.22749.

26. Dzik-Jurasz A, Domenig C, George M, Wolber J, Padhani A, Brown G, et al. Diffusion MRI for prediction of response of rectal cancer to chemoradiation. Lancet. 2002;360(9329):307-8. https://doi.org/10.1016/S0140-6736(02)09520-X.

27. Cai G, Xu Y, Zhu J, Gu WL, Zhang S, Ma XJ, et al. Diffusion-weighted magnetic resonance imaging for predicting the response of rectal cancer to neoadjuvant concurrent chemoradiation. World J Gastroenterol. 2013;19(33): 5520-7. https://doi.org/10.3748/wjg.v19.i33.5520.

28. Sun YS, Zhang XP, Tang L, Ji JF, Gu J, Cai Y, et al. Locally advanced rectal carcinoma treated with preoperative chemotherapy and radiation therapy: preliminary analysis of diffusion-weighted MR imaging for early detection of tumor histopathologic downstaging. Radiology. 2010;254(1):170-8. https:// doi.org/10.1148/radiol.2541082230.

\section{Publisher's Note}

Springer Nature remains neutral with regard to jurisdictional claims in published maps and institutional affiliations.

\section{Ready to submit your research? Choose BMC and benefit from:}

- fast, convenient online submission

- thorough peer review by experienced researchers in your field

- rapid publication on acceptance

- support for research data, including large and complex data types

- gold Open Access which fosters wider collaboration and increased citations

- maximum visibility for your research: over $100 \mathrm{M}$ website views per year

At $\mathrm{BMC}$, research is always in progress.

Learn more biomedcentral.com/submissions 